\title{
Khellaf Rebbas
}

\section{Première observation de Physalis acutifolia (Solanaceae) dans la région de M'Sila (Algérie), nouvelle pour l'Afrique du nord}

\begin{abstract}
Rebbas, K.: Première observation de Physalis acutifolia (Solanaceae) dans la région de M'Sila (Algérie), nouvelle pour l'Afrique du nord. — Fl. Medit. 28: 21-25. 2018. — ISSN: 1120-4052 printed, 2240-4538 online.

Physalis acutifolia (Solanaceae), native to the southwestern United States, California Texas, and northern Mexico, has been observed in many localities in the M'Sila region, central north Algeria. Distribution and ecological notes are also provided. This is the first report of this species in N Africa.
\end{abstract}

Key words: xenophytes, weeds, North Africa, medicinal plants.

\section{Introduction}

Le genre Physalis comprend un certain nombre d'espèces comestibles et ornementales d'importance commerciale. Sa haute valeur nutritionnelle et ses propriétés médicinales potentielles entraînent un intérêt commercial accru pour les produits de ce genre dans le monde entier (Wei \& al. 2012).

Ce genre contient plus de 90 espèces dont 35 dans la région de Mexique (Cuevas-Arias $\&$ al. 2008). Physalis acutifolia (Miers) Sandw. (= Saracha acutifolia Miers, Physalis wrightii Gray) est originaire des États-Unis, de la Californie au Texas, et au nord du Mexique, où il peut être trouvé dans de nombreux types d'habitats, y compris les zones perturbées. C'est parfois une mauvaise herbe quand elle se développe dans les champs agricoles, mais elle n'est généralement pas envahissante dans l'habitat sauvage.

En Afrique du nord, la plante n'est pas citée dans l'index de Dobignard \& Chatelain (2010-2013). De même aucune flore ou catalogue d'Algérie (Battandier 1888-1890; Battandier \& Trabut 1905; Maire 1952-1987; Quézel \& Santa 1962-1963; Ozenda 1983) ni même de Tunisie (Bonnet \& Barrate 1896; Pottier-Alapetite 1979-1981; Le Floc'h \& al. 2010; Guittonneau 2011), ni aussi au Maroc (Fennane \& Ibn Tattou 1998, 2005; Fennane $\&$ al. 2007) ne signale ce taxon. Cette plante n'est pas signalée dans l'Euro+Med PlantBase (Euro+Med 2006-) et pas même dans les plus récents contributions sur les espèces étrangères dans les territoires voisins (Celesti Grapow \& al. 2016). 
L'index de Dobignard \& Chatelain (2010-2013) a cité 5 espèces de Physalis: P. alkekengi L. et $P$. viscosa L. au Maroc, $P$. angulata L. en Mauritanie, Maroc, Egypte, P. ixocarpa Hornem. en Egypte, $P$. peruviana L. en Madère, Mauritanie, Maroc.

En Algérie, Munby (1847) a signalé Physalis somnifera L., sur les décombres autour d'Alger, au cimetière des Juifs à Bab-el-Oued.

\section{Description botanique et répartition géographique}

Physalis acutifolia (Miers) Sandw. est une plante annuelle, avectiges et branches étalées et ascendantes, pubescentes et légèrement pubescentes sur les bords et les nervures des feuilles, plus denses sur les nouvelles pousses, les tiges atteignant $50 \mathrm{~cm}$ de long; poils atteignant $1 \mathrm{~mm}$ de long, non glanduleux. Feuilles elliptiques, souvent étroites, parfois lancéolées ou ovales, de 4-12,5 cm de long, de 1-5 cm de large, 1,8-6 fois plus longues que larges; base aiguë à acuminée souvent obliquement ainsi; apex aigu à acuminé; bords dentés à incisés ou dentés. Fleurs de 0,7-1,2 cm de long; pédoncule de 1,2-3 cm de long, habituellement environ 2-3 fois plus long que la fleur; calice de 0,3-0,5 cm de long, les lobes étant aussi longs que le tube; corolle blanc jaunâtre, parfois avec centre obscurci orange jaunâtre, 1-2,2 cm de large; anthères jaunes, 3-4 mm de long, environ 3-4 fois plus longues que larges. Le calice ovoïde, 1,2-2,3 cm de long, 1-2 cm de large, baie de 0,6-1,3 cm de diamètre; graines lenticulaires, 2-2.2 $\mathrm{mm}$ de large. Floraison et fructification, JuilletSeptembre (Landrum \& al. 2013)

Dans la région de M'Sila, cette solanacée a été observée dans 5 stations localisées dans des champs de culture du piment (Tableau 1, Fig. 1 et 2).

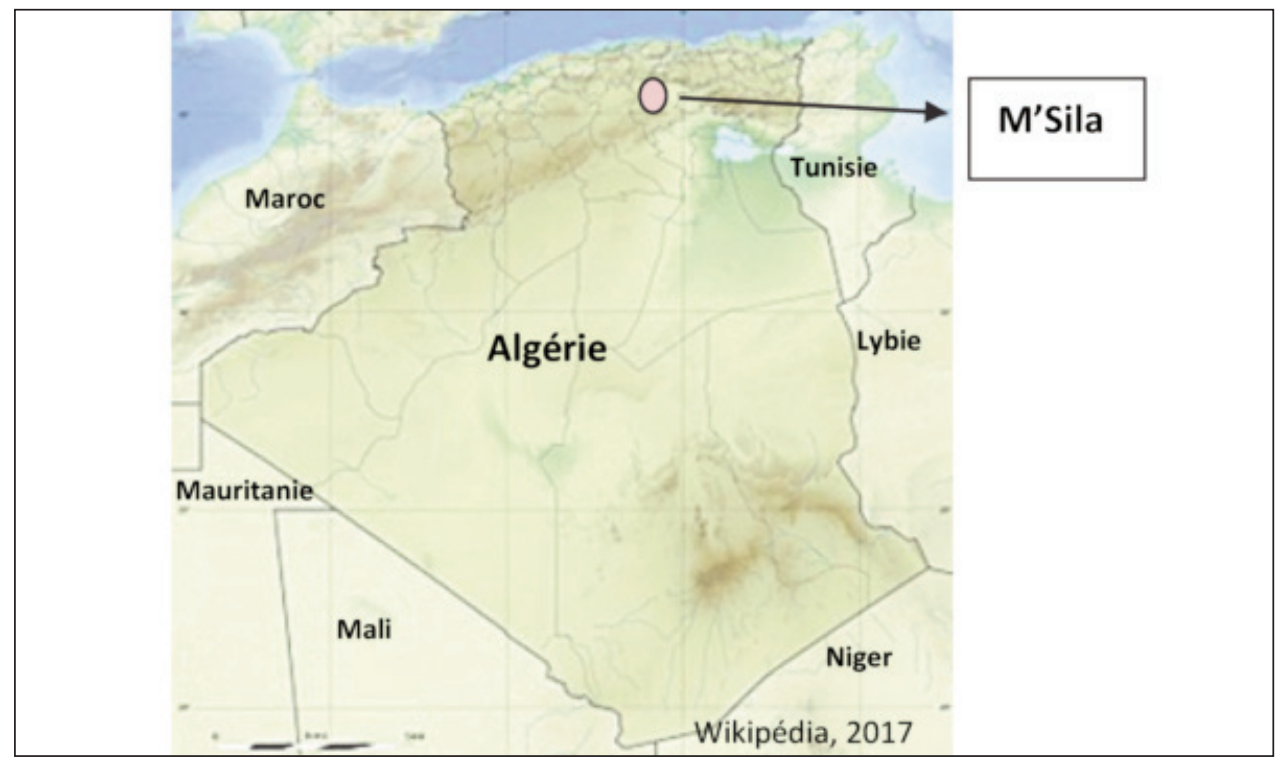

Fig. 1. Localisation géographique de la région de M'Sila. 
Tab. 1. Localisation des stations d'observation de P. acutifolia dans la région de M'Sila.

\begin{tabular}{|l|c|c|c|c|c|}
\hline Station & Nbre de plantes & N & E & Altitude (m) & Date \\
\hline Lamarate & 3 & $35^{\circ} 32^{\prime} 58^{\prime \prime}$ & $04^{\circ} 29^{\prime} 21^{\prime \prime}$ & 380 & $18 / 8 / 2017$ \\
\hline Saïda & 7 & $35^{\circ} 35^{\prime} 12^{\prime \prime}$ & $04^{\circ} 29^{\prime} 45^{\prime \prime}$ & 376 & $18 / 8 / 2017$ \\
\hline OuledMadi & 4 & $35^{\circ} 36^{\prime} 25^{\prime \prime}$ & $04^{\circ} 30^{\prime} 16^{\prime \prime}$ & 361 & $18 / 8 / 2017$ \\
\hline El Beida & 3 & $35^{\circ} 37^{\prime} 17^{\prime \prime}$ & $04^{\circ} 29^{\prime} 39^{\prime \prime}$ & 424 & $18 / 8 / 2017$ \\
\hline Maaza & 2 & $35^{\circ} 52^{\prime} 16^{\prime \prime}$ & $04^{\circ} 23^{\prime} 45^{\prime \prime}$ & 696 & $15 / 9 / 2017$ \\
\hline
\end{tabular}

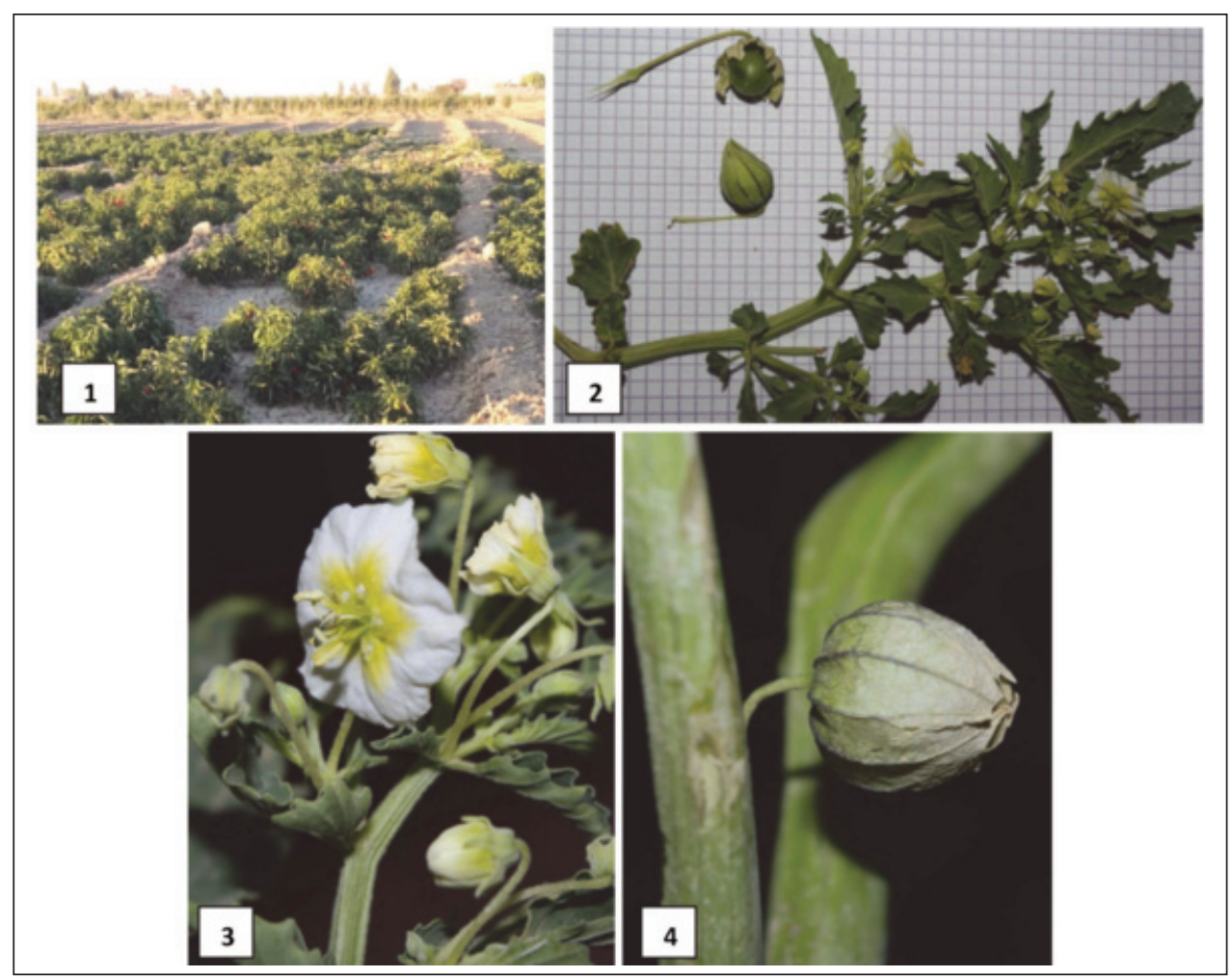

Fig. 2. Physalis acutifolia (1. Habitat: champ de culture du piment; 2. Port; 3. Fleur; 4. Fruit, photos: K. Rebbas, 18.8.2017).

\section{Intérêts et Activités pharmacologiques}

Physalis L. est un genre américain qui a une utilisation alimentaire enracinée dans l'alimentation quotidienne de nombreuses régions géographiques du Mexique, où les espèces $P$. philadelphica et $P$. angulatas ont cultivées, ainsi que la récolte des fruits d'au moins une douzaine d'espèces sauvages. Les preuves de la valeur nutritive et fonctionnelle des fruits sont passées en revue, citant le contenu de certaines espèces en fibres, minéraux, protéines, 
lipides solubles et sucres, ainsi que les vitamines $\mathrm{A}$ et $\mathrm{C}$, certains principes phytochimiques bioactifs aux propriétés antioxydantes, anti-cancer, anti-inflammatoire et antibiotique, entre autres. Ces propriétés font des tomates à coque des modèles biologiques de grand intérêt pour les industries agricoles et pharmaceutiques (Vargas-Ponce 2015).

Les plantes de Physalis sont aussi largement répandues dans toute l'Inde, la Chine et d'autres pays subtropicaux. Les alcalö̈des, les flavonoïdes et les withanolides sont les constituants primaires des espèces de Physalis principalement considérées pour être responsables de ses actions thérapeutiques larges. En dehors de l'activité analgésique, ces plantes sont utilisées pour traiter le cancer, la leucémie, l'hépatite, ayant des propriétés comme hépatoprotecteur, diurétique, anti-ulcère, anti-microbienne, anti-oxydante, antiinflammatoire, une activité protectrice rénale (Jyothibasu \&Venkata 2015). D'autres espèces de même genre comme Physalis somnifera L. (Withania somnifera (L.) Dunal) a été utilisée pendant des siècles dans la médecine traditionnelle indienne (Mirjalili \& al. 2009; Pandit \& al. 2013). Au Yémen, où il est connu sous le nom d'ubab, les feuilles séchées sont broyées en une poudre à partir de laquelle une pâte est fabriquée et utilisée pour les brûlures et les plaies (Hehmeyer \& Schönig 2012).

\section{Conclusion}

Physalis acutifolia, ainsi que pour la flore d'Afrique du Nord, est une nouveauté pour toute la région méditerranéenne. Cette découverte enrichit davantage la flore vasculaire algérienne et elle constitue un ajout important pour la flore médicinale du pays.

Elle est fort probable que la population de P. acutifolia provient de graines introduites au niveau des champs de culture du piment.

\section{Remerciements}

Je tiens à remercier vivement Marcus Joseph A.(Lady Bird Johnson Wildflower Center, Texas) qui m'a aidé à identifier cette solanacée et le (s) relecteur(s) anonyme(s) pour les diverses améliorations apportées pour ce travail.

\section{Références}

Battandier, J. A. 1888-1890: Flore d'Algérie: Ancienne flore d'Alger transformée (Dicotylédones). - Alger.

— \& Trabut, L. C. 1905: Flore analytique et synoptique de l'Algérie et de la Tunisie. - Alger.

Bonnet, E. \& Barratte, G. 1896: Catalogue raisonné des plantes de la Tunisie. - Paris.

Celesti-Grapow, L., Bassi, L., Brundu, G., Camarda, I., Carli, E., D’Auria, G, Del Guacchio, E., Domina, G., Ferretti, G., Foggi, B., Lazzaro, L., Mazzola, P., Peccenini, S., Pretto, F., Stinca, A. \& Blasi, C. 2016: Plant invasions on small Mediterranean islands: An overview. - P1. Biosyst. 150(2): 1119-1133. doi: 10.1080/11263504.2016.1218974

Cuevas-Arias, C. T., Vargas, O. \& Rodrigues, A. 2008: Solanaceae diversity in the state of Jalisco, Mexico. - Rev. Mexicana Biodiv. 79: 67-79.

Dobignard, A. \& Chatelain, C. 2010/2013: Index synonymique de la flore d'Afrique du Nord. Genève. 
Euro+Med (2006-): Euro+Med PlantBase - the information resource for Euro-Mediterranean plant diversity. Published on the Internet http://ww2.bgbm.org/EuroPlusMed/ [dernier accès 05/01/2018].

Fennane, M. \& Ibn Tattou, M. 1998: Catalogue des plantes vasculaires rares, menacées ou endémiques du Maroc. - Bocconea 8: 5-243.

— \& - 2005: Flore vasculaire du Maroc, inventaire et chorologie. - Trav. Inst. Sci. Univ. Mohammed V, Sér. Bot. 37: 1-202.

— \& - , Ouyahya, A. \& El Oualidi, J. 2007: Flore pratique du Maroc. - Trav. Inst. Sci. Univ. Mohammed V, Sér. Bot. 38:1-636.

Guittonneau, G. G. 2011: La flore et la végétation de la Tunisie méridionale. - Voy. Etud. Soc. Bot. France (27 mars -3 avril 2008): 283-358 - http://www.biusante.parisdescartes.fr/ sbf/cr_tunisie.pdf

Hehmeyer, I. \& Schönig, H. 2012: Herbal Medicine in Yemen: Traditional Knowledge and Practice, and Their Value for Today's World”. - Islamic Hist. Civilization 96: 1-249.

Jyothibasu, T. \& Venkataramana, K. 2015: Pharmacological review on Physalis species: a potential herbal cure-all. - World J. Pharmac. Res. 4(2): 247-256.

Le Floc'h, E., Boulos, L. \& Véla, E. 2010: Catalogue synonymique commenté de la flore de Tunisie. - Tunis.

Landrum, L. R., Barber, A., Barron, K., Coburn, F. S., Sanderford, K. \& Setaro, D. 2013 : Vascular Plants of Arizona: SolanaceaePart Four : Physalis L. and Quincula Raf. Ground Cherry, Tomatillo. - Canotia 9: 1-12.

Maire, R. 1952-1987: Flore de l'Afrique du Nord (Maroc, Algérie, Tunisie, Tripolitaine, Cyrénaïque et Sahara), 1-16. - Paris.

Mirjalili, M. H., Moyano, E., Bonfill, M., Cusido, R. M. \& Palazón, J. 2009: Steroidal Lactones from Withania somnifera, an Ancient Plant for Novel Medicine. - Molecules 14(7): 2373-2393. doi : 10.3390/molecules14072373

Munby, G. 1847: Flore de l'Algérie ou catalogue des plantes indigènes. - Paris.

Ozenda, P. 1983: Flore du Sahara. - Paris.

Pandit, S., Chang, K. W. \& Jeon, J. G. 2013: Effects of Withania somnifera on the growth and virulence properties of Streptococcus mutans and Streptococcus sobrinus at sub-MIC levels. Anaerobe 19: 1-8.

Pottier-Alapetite, M. 1979-1981: Flore de la Tunisie, Angiospermes-Dicotylédones, 1-2. - Tunis.

Quézel, P. \& Santa, S. 1962-1963: Nouvelle flore de l'Algérie et des régions désertiques méridionales, 1-2. - Paris.

Vargas-Ponce, O., Valdivia-Mares, L. E. \& Sánchez-Martínez, J. 2015: Potencial alimentacio de Los tomates de Cascara (Physalis spp.) de México - Dietary potential of husk tomatoes (Physalis spp.) in Mexico. - Agro 8(1): 9-17.

Wei, J., Hu, X., Yang, J. \&Yang, W. 2012: Identification of Single-Copy Orthologous Genes between Physalis and Solanum lycopersicum and Analysis of Genetic Diversity in Physalis Using Molecular Markers. - PLoS One 7(11): e50164. doi: 10.1371/journal.pone.0050164

Adresse de l'auteur:

Khellaf Rebbas,

Département des Sciences de la Nature et de la Vie, Faculté des Sciences, Université Mohamed Boudiaf de M'Sila, 28 000, Algérie. Courriel: rebbaskhellaf@yahoo.fr 
